\title{
Insulating phases of two-dimensional electrons in high Landau levels: Observation of sharp thresholds to conduction
}

\author{
K. B. Cooper, M. P. Lilly, and J. P. Eisenstein \\ California Institute of Technology, Pasadena, California 91125 \\ L. N. Pfeiffer and K. W. West \\ Bell Laboratories, Lucent Technologies, Murray Hill, New Jersey 07974
}

(Received 25 June 1999)

\begin{abstract}
The intriguing re-entrant integer quantized Hall states recently discovered in high Landau levels of highmobility 2D electron systems are found to exhibit extremely nonlinear transport. At small currents these states reflect insulating behavior of the electrons in the uppermost Landau level. At larger currents, however, a discontinuous and hysteretic transition to a conducting state is observed. These phenomena, found only in very narrow magnetic field ranges, are suggestive of the depinning of a charge density wave state, but other explanations can also be constructed. [S0163-1829(99)51740-2]
\end{abstract}

Two-dimensional electron systems (2DES) in strong magnetic fields have proven to be a remarkably rich laboratory for many-body physics. ${ }^{1}$ The continuing improvements in the techniques for creating 2DES in semiconductor heterostructures have been paralleled by a steady stream of discoveries of novel electron correlation phenomena. While the fractional quantum Hall effect in the lowest $(N=0)$ Landau level (LL) is the best known of these, there has been a recent realization that interactions among electrons in the excited LLs can give rise to whole new classes of many-body phenomena. For example, recent transport measurements ${ }^{2,3}$ have revealed huge and unexpected anisotropies of the resistivity of the 2DES when the third and higher $(N \geqslant 2)$ LLs are half filled. These anisotropies are not seen in the lowest two LLs and appear only at very low temperatures and in the highest quality samples. The observations are in qualitative agreement with earlier theoretical suggestions of unidirectional charge density wave (CDW) ground states ("stripe phases") in the half-filled $N \geqslant 2$ LLs. ${ }^{4,5}$ More recent theoretical work, ${ }^{6-9}$ going beyond the Hartree-Fock approximation, has lent support to the stripe phase picture, albeit with possibly important modifications due to quantum fluctuations.

The experiments in high Landau levels also reveal remarkable phenomena away from half filling, in the flanks of the LLs. In this regime both Lilly et l. $^{2}$ and Du et $a .^{3}$ reported the resistivity to be essentially isotropic and to fall to zero in narrow regions of magnetic field near $1 / 4$ and $3 / 4$ filling of the LLs. In these regions the Hall resistance is found to be accurately quantized but, quite surprisingly, at the value of the adjacent integer quantum Hall plateaus. These re-entrant integer quantum Hall effect (RIQHE) states, which have only been found in the $N \geqslant 2$ LLs, suggest the existence of insulating phases of the electrons in the uppermost LL. In this paper we report the observation of a discontinuous transition from the insulating state to a conducting one when large electric fields are applied. This transition is found to be hysteretic and extremely temperature and magnetic field dependent. The results are suggestive of the depinning of CDWs, ${ }^{10}$ but they are also reminiscent of quantum Hall breakdown phenomena. ${ }^{11}$
The samples used in this investigation are modulationdoped GaAs/AlGaAs heterostructures grown by molecular beam epitaxy. Data from two samples (A and B) are discussed here. Sample A exhibits a 2D electron density of $n_{s}$ $=2.7 \times 10^{11} \mathrm{~cm}^{-2}$ and a low temperature mobility of 1.1 $\times 10^{7} \mathrm{~cm}^{2} /$ Vs. Sample B has a density of $n_{s}=2.1$ $\times 10^{11} \mathrm{~cm}^{-2}$ and a mobility of $1.6 \times 10^{7} \mathrm{~cm}^{2} / \mathrm{Vs}$. These parameters are determined after brief illumination with red light at low temperature. Each sample consists of a square cleaved from its parent wafer along $\langle 110\rangle$ and $\langle 1 \overline{1} 0\rangle$ crystal directions. Sample A is $5 \times 5 \mathrm{~mm}^{2}$ while sample B is 2.5 $\times 2.5 \mathrm{~mm}^{2}$. Eight indium ohmic contacts are placed at the corners and midpoints of the sides of the square. Longitudinal resistance measurements are performed by injecting and withdrawing current from two midpoint contacts on opposite sides of the square sample while recording the voltage difference between two corner contacts. Both direct and low frequency alternating currents have been employed in these experiments.

Figure 1(a) shows transport data from sample A at $T$ $=50 \mathrm{mK}$ taken using an excitation current of $10 \mathrm{nA}$ at 13 Hz. The data cover magnetic fields $B$ between LL filling fractions $\nu \equiv h n_{s} / e B=5$ and $\nu=4$. In this range the Fermi level of the 2DES is in the lower spin branch of the $N=2$ LL. The solid curves are the longitudinal and Hall resistances, $R_{x x}$ and $R_{x y}$, observed for net current flow along the $\langle 1 \overline{1} 0\rangle$ direction. The dotted curve is the longitudinal resistance $R_{y y}$ resulting from net current flow along the $\langle 110\rangle$ direction. The giant anisotropy of the resistance near half filling of high Landau levels reported earlier ${ }^{2,3}$ is clearly evident. This transport anisotropy, which is not seen in the $N$ $=0$ or $1 \mathrm{LL}$, disappears above about $150 \mathrm{mK}$. In the same field range no quantized plateaus appear in $R_{x y}$. The shoulders visible in Fig. 1(a) on either side of $\nu=9 / 2$ are not nascent plateaus. They shift, and ultimately disappear, as the temperature is lowered to $T=25 \mathrm{mK}$.

The data in Fig. 1(a) also show that the resistance becomes approximately isotropic in the flanks of the Landau level. Furthermore, there are clearly defined regions of mag- 


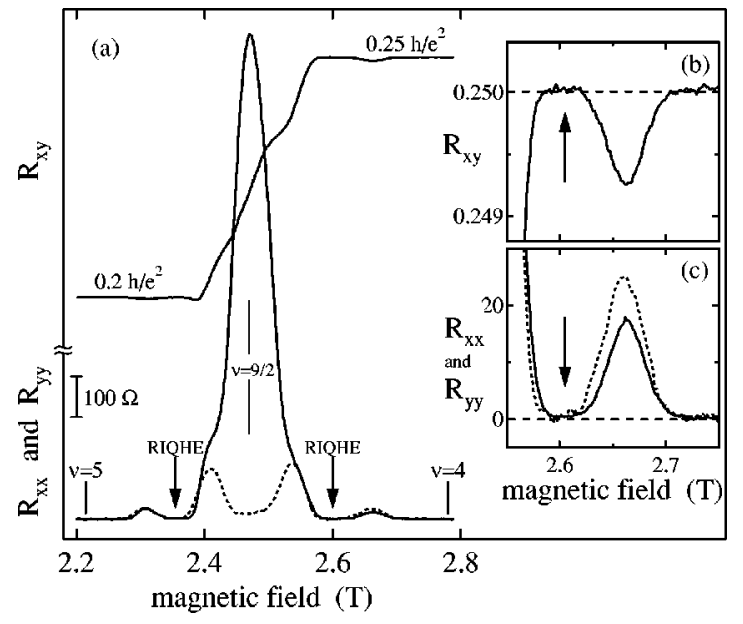

FIG. 1. (a) Longitudinal (solid line: $R_{x x}$, dotted line: $R_{y y}$ ) and Hall $\left(R_{x y}\right)$ resistance of sample A in the $N=2$ Landau level at $T$ $=50 \mathrm{mK}$. Arrows indicate the positions of the re-entrant integer quantum Hall effect (RIQHE) states. Insets (b) and (c) magnify the RIQHE region.

netic field (indicated by arrows) on each side of $\nu=9 / 2$ in which both $R_{x x}$ and $R_{y y}$ have dropped (in an approximately thermally activated manner ${ }^{3}$ ) to vanishingly small values. These features, which suggest the existence of fractional quantized Hall states, occur near filling factors $\nu \approx 4 \frac{1}{4}$ and $4 \frac{3}{4}$. Measurements of the Hall resistance $R_{x y}$ do indeed show quantization, but at the value of the nearest integer quantized Hall plateau. Figures 1(b) and 1(c) demonstrate this via magnified views of the resistances. The data show that the RIQHE states are separated from the main integer states by narrow regions of field in which $R_{x x}$ (and $R_{y y}$ ) is non-zero and $R_{x y}$ is not quantized. ${ }^{2,3}$ In common with the transport anisotropies at half filling, these intriguing quantum hall effect (QHE) features are seen in several high LLs but they are conspicuously absent in the $N=1$ and 0 level.

In the standard picture of the integer QHE, the finite width of Hall plateaus and zero resistance states is attributed to the localization, via disorder, of the quasiparticles in the system. Moving away from integer filling increases the density and localization length of these quasiparticles. When they eventually delocalize, the quantization of $R_{x y}$ is lost and $R_{x x}$ becomes non-zero. The data in Fig. 1 show, quite remarkably, that these same quasiparticles apparently localize again at slightly higher densities where the RIQHE forms. This result cannot be readily understood in the standard single-particle localization picture of the integer QHE but instead suggests that these new insulating phases in high LLs are critically dependent upon electron-electron interactions. In the absence of disorder when the uppermost LL is just beginning to fill, interactions should lead to Wigner crystallization. With weak disorder this crystal would be pinned and therefore non-conducting. As the level is further filled, the Wigner crystal presumably melts. Interestingly, recent theoretical works predict the existence, in high LLs, of additional ordered phases ("bubbles" 4,5 and "stripe crystals" 6 ) between this Wigner crystal at the very edge of the LL and the unusual anisotropic phases at half filling. Conceivably, the insulating behavior that we observe in the RIQHE reflects the pinning of one of these new phases.

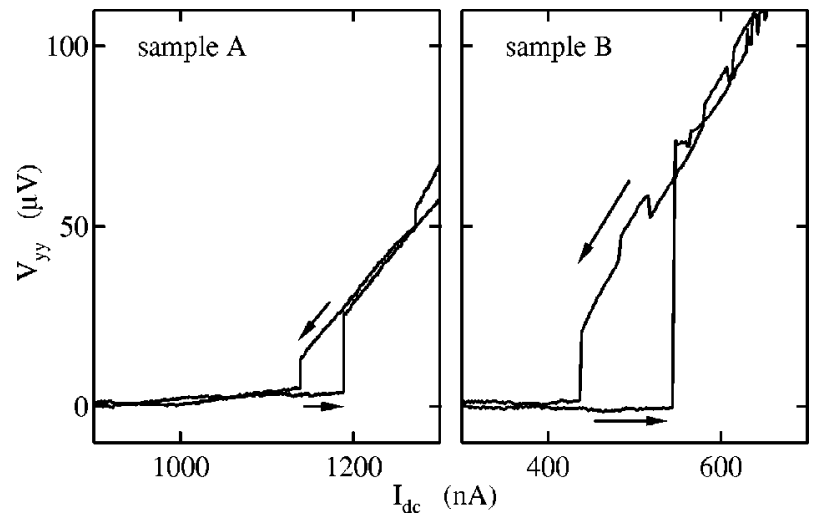

FIG. 2. Discontinuous current-voltage characteristics observed at $T=25 \mathrm{mK}$ in the center of the re-entrant integer quantum Hall effect near $\nu \approx 4 \frac{1}{4}$. Arrows denote direction of current sweep.

To examine this possibility, we have performed dc current-voltage $(I-V)$ studies of the RIQHE states. Figure 2 shows representative results from both samples. The data shown come from the RIQHE region around $\nu \approx 4 \frac{1}{4}$ at $T$ $=25 \mathrm{mK}$. The dc current (along $\langle 110\rangle)$ is slowly swept up from zero to as much as $1500 \mathrm{nA}$ and then swept down again while the longitudinal voltage is recorded. In both samples a sharply defined threshold current is found where the voltage jumps discontinuously. The transitions are hysteretic; the threshold current is almost always larger when the current is swept up than when it is swept down. Just above threshold the voltage rises approximately linearly with current, although additional small features can be seen. For sample A the threshold is around $1200 \mathrm{nA}$; for sample $\mathrm{B}$ it is around $500 \mathrm{nA}$. The precise threshold current also depends sensitively upon the temperature and magnetic field within the RIQHE region. No qualitative dependence of the thresholds upon the current flow direction or voltage probe configuration has been found, although quantitative variations are indeed observed.

These sharp onsets of conduction are only seen in the immediate vicinity of the RIQHE states. Figure 3 shows a sampling of $I-V$ curves from sample B (for clarity only sweeps with increasing current are shown). These data are taken at equally spaced magnetic fields $(\Delta B=13 \mathrm{mT})$ from the low field side of the RIQHE near $\nu \approx 4 \frac{1}{4}$ to the center the $\nu=4$ integer QHE state. Also shown is the conventional resistance $R_{y y}$ measured with a $10 \mathrm{nA}$ ac current excitation. It is clear from the figure that the discontinuous jumps in the dc $I-V$ curves are only seen in the RIQHE state. Deep inside the ordinary $\nu=4$ QHE state $V_{y y}$ remains zero, even out to much higher currents than shown in the figure. Outside of the RIQHE, at most weak and smoothly varying non-linearities are found. Essentially the same results are found in the vicinity of the $\nu \approx 4 \frac{3}{4}$ RIQHE. Sample A shows non-linear behavior that is quite similar to sample B, albeit with higher threshold currents.

The qualitative difference between the $I-V$ curves observed in the RIQHE and the conventional integer quantum Hall effect (IQHE) state is particularly striking when the temperature dependence of the longitudinal resistance separating these regions is considered. The small peaks in $R_{y y}$ and $R_{x x}$ shrink as the temperature is reduced. In sample $\mathrm{B}$ they remain visible down to below $\mathrm{T}=25 \mathrm{mK}$ but in sample 


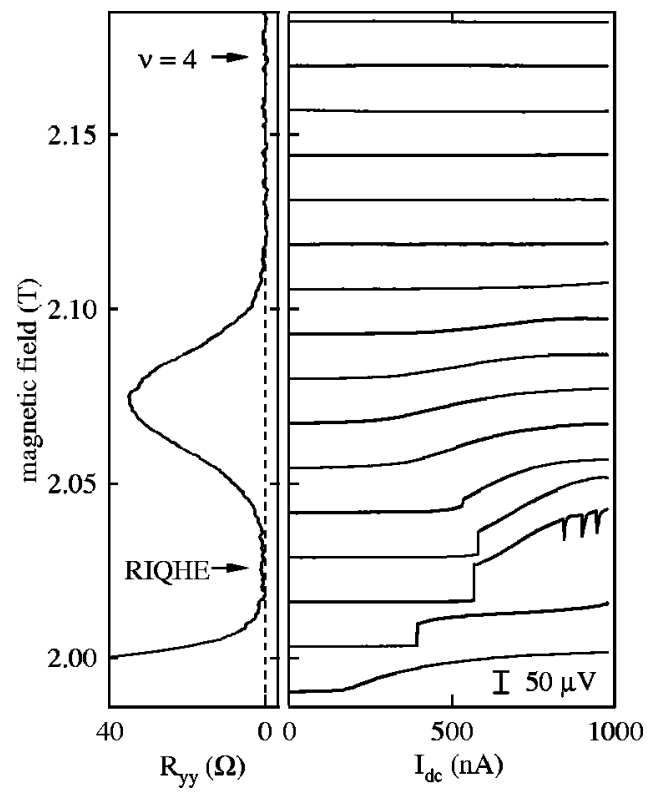

FIG. 3. Demonstration that $I-V$ discontinuities are confined to the re-entrant integer quantum Hall effect. Left panel: $R_{y y}$ vs $B$ for sample B. Right panel: Family of $I-V$ curves, each vertically displaced to coincide with its magnetic field location on the resistance plot in the left panel.

A, which has lower mobility, they essentially vanish. In spite of this, the discontinuities in the $I-V$ curves are still found only in the narrow field range defined as the re-entrant IQHE state via the resistance observed at higher temperature.

The dc currents employed for Fig. 3 are large. Although the majority of that current flows in the four edge channels of the $N=0$ and 1 Landau levels which lie below the Fermi level in the bulk of the sample, the possibility of electron heating must be considered. Figure 4 shows that this is, perhaps surprisingly, not a serious problem. The $I-V$ curves in the vicinity of threshold are quite sensitive to temperature down to below $25 \mathrm{mK}$. As the temperature is raised, the conduction threshold shifts to lower dc currents and disap-

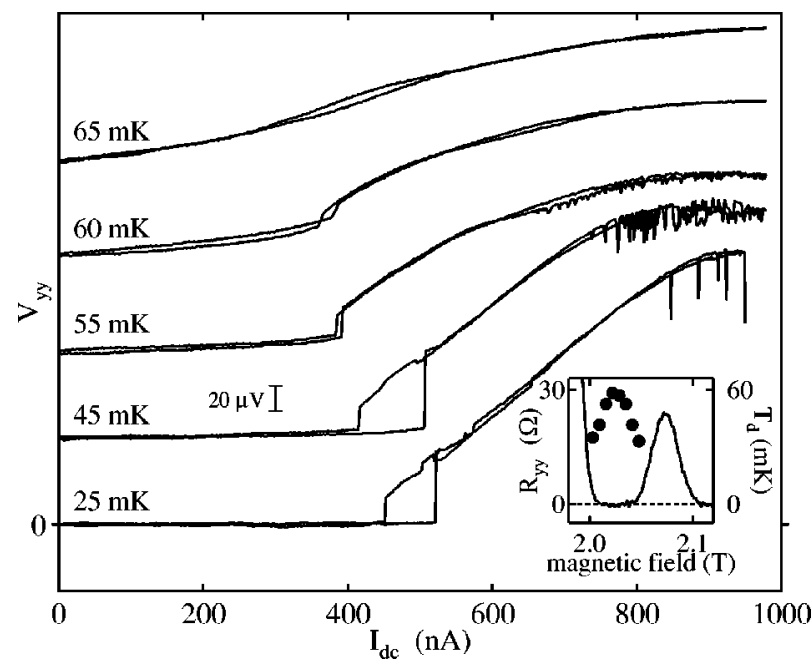

FIG. 4. $I-V$ curves at several temperatures for sample B at $B$ $=2.02$ Tesla. Curves are offset vertically for clarity. Inset: The step disappearance temperature $T_{d}$ (dots) and $R_{y y}$ at $T=25 \mathrm{mK}$ (solid line). pears abruptly. In the inset to Fig. 4 , the temperature $T_{d}$ at which the discontinuities disappear is plotted as a function of magnetic field. $T_{d}$ is largest $(\sim 60 \mathrm{mK})$ at the center of the RIQHE, and falls off rapidly on either side.

Also apparent in Fig. 4 is the presence of noise in the conducting state above threshold. Downward spikes in the voltage are observed in the vicinity (magnetic field and temperature) of where the discontinuity in the $I-V$ is observed. When the temperature and dc current are fixed, the spikes occur almost periodically. The period, typically 1 to $40 \mathrm{sec}-$ onds depending on temperature, decreases as $T$ approaches $T_{d}$. Above $T_{d}$ no voltage spikes are seen. No spikes have been found, at any temperature, outside the RIQHE region.

These non-linear transport phenomena are suggestive of the depinning and sliding transport of CDWs. ${ }^{10}$ In the present case, the current flowing through the sample (via the filled Landau levels beneath the Fermi level) produces a Hall electric field transverse to the current. This field exerts a force on the localized electrons in the uppermost Landau level. For small currents, this force is insufficient to delocalize these carriers and the longitudinal resistance of the sample remains zero. As the current increases these electrons eventually delocalize and the resistances become non-zero. If the electrons are individually localized by a random disorder potential, a gradual onset of conduction is expected. If, on the other hand, large collections of highly correlated electrons are pinned at a small number of sites, a much more abrupt transition to conduction is anticipated. Our data reveal both kinds of behavior but only in the RIQHE are sharp onsets of conduction observed.

Similar ideas have been applied in the past to the 2DES at very high magnetic fields where insulating behavior is observed $^{12}$ in the lowest Landau level $(\nu \lesssim 0.2$ in clean electron systems). Non-linear transport measurements ${ }^{13-16}$ have indicated that threshold fields (of varying sharpness) exist in this regime and many have interpreted them as evidence for
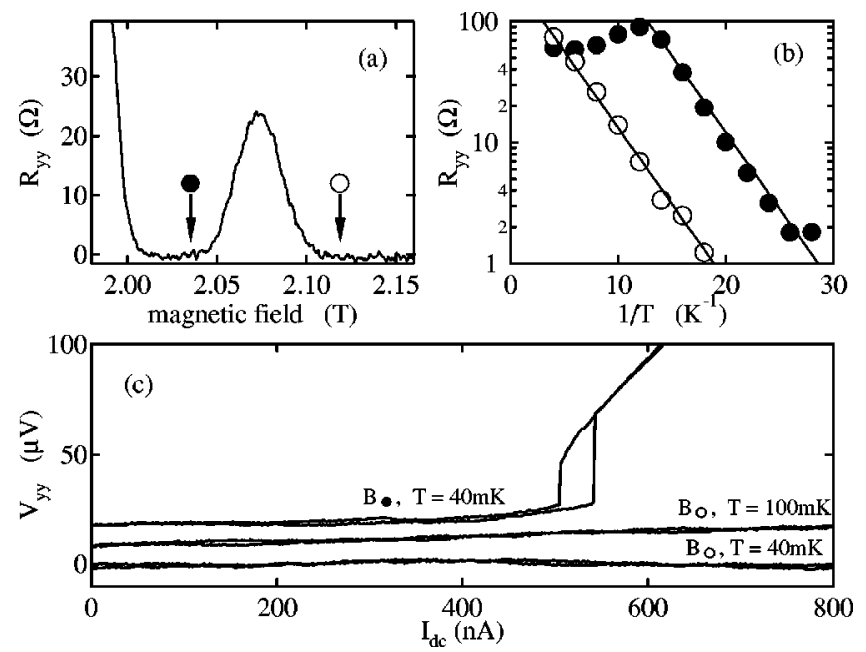

FIG. 5. Comparison of transport data just inside and just outside the re-entrant integer quantum Hall effect (RIQHE) state in sample B. Panel (a) indicates the magnetic fields where $I-V$ curves were taken. In (b), an Arrhenius plot shows the temperature dependence for the two fields, with lines to guide the eye. In (c), I- $V$ curves (offset for clarity) show a discontinuous threshold only in the RIQHE state (top curve) and not at the other field location (e.g., middle and bottom curves). 
the depinning of an isotropic Wigner crystal. In our case, where the insulating electrons are those in a partially filled high Landau level, the spectrum of possible pinned correlated states is broader and includes bubble ${ }^{4,5}$ and stripe crystal $^{6}$ phases.

The non-linearity phenomena reported here are also qualitatively similar to what has been seen in studies of the breakdown of the integer quantized Hall effect. Discontinuous $I-V$ curves, hysteresis, and excess noise near the onset of conduction have all been reported. ${ }^{11}$ As Jiang et al. ${ }^{16}$ emphasize in their study of the insulating behavior of 2D electron systems at very low filling factor, the observation of electric field thresholds in the insulating state may be related to a thermal breakdown process, and not the sliding of an underlying crystalline state. While thermal run-away models ${ }^{11,17}$ depend upon many factors, key among them is simply the temperature dependence of the longitudinal resistivity. To investigate the relevance of such models to the RIQHE, we compare the temperature dependence of the resistance $R_{y y}$ at two filling factors, one inside the RIQHE which shows a sharp conduction onset, and one outside it which does not. These two filling factors, indicated in Fig. 5(a), are located symmetrically about the small peak in $R_{y y}$ which separates the RIQHE from the conventional $\nu=4$ QHE. At both locations temperature dependence (Fig. 5(b)) of the resistance behaves in an activated manner: $R_{y y}=R_{0} \exp \left(-E_{A} / T\right)$. While the measured activation energies in the two cases are comparable $\left(E_{A} \sim 0.3 \mathrm{~K}\right)$, the prefactor $R_{0}$ is about 17 times larger at the point inside the RIQHE than at the point outside it. At $T=40 \mathrm{mK}, R_{y y}$ is small but non-zero at the RIQHE point and yet a sharp jump in the $I-V$ curve was still visible (Fig. 5(c)). In contrast, at no temperature were discontinuous (or even sharp) features observed at the other field location, a mere $70 \mathrm{mT}$ away. This was true regardless of whether the actual $R_{y y}$ value was smaller, equal to, or larger than the value inside the RIQHE where a sharp conduction onset was observed. While these observations do not completely eliminate thermal run-away models of the $I-V$ discontinuities in the RIQHE, they do suggest that their origin lies elsewhere.

In conclusion, we report transport measurements of insulating states on the flanks of the $N=2$ and higher LLs. In these regions the longitudinal resistance vanishes and the Hall resistance becomes quantized at the nearby IQHE value. The distinction of these RIQHE states from the conventional IQHE suggests that electron correlations are very important in their structure. Measurements of current-voltage characteristics reveal discontinuous and hysteretic transitions between insulating and conducting phases of the electrons in the uppermost LL. These dramatic non-linearities have only been found within the RIQHE and at very low temperatures $(T<60 \mathrm{mK})$. These findings are highly suggestive of depinning of charge density waves, but other mechanisms may yet prove responsible.

This work was supported by the National Science Foundation and the Department of Energy.
${ }^{1}$ For recent reviews, see Perspectives in Quantum Hall Effects, edited by S. Das Sarma and A. Pinczuk (John Wiley, New York, 1997).

${ }^{2}$ M.P. Lilly et al., Phys. Rev. Lett. 82, 394 (1999).

${ }^{3}$ R.R. Du et al., Solid State Commun. 109, 389 (1999).

${ }^{4}$ A.A. Koulakov, M.M. Fogler, and B.I. Shklovskii, Phys. Rev. Lett. 76, 499 (1996); Phys. Rev. B 54, 1853 (1996); 55, 9326 (1997).

${ }^{5}$ R. Moessner and J. Chalker, Phys. Rev. B 54, 5006 (1996).

${ }^{6}$ E. Fradkin and S. Kivelson, Phys. Rev. B 59, 8065 (1999); E. Fradkin, S. Kivelson, E. Manousakis, and K. Nho, cond-mat/ 9906064 (unpublished).

${ }^{7}$ H. Fertig, Phys. Rev. Lett. 82, 3693 (1999).

${ }^{8}$ E.H. Rezayi, F.D.M. Haldane, and K. Yang, Phys. Rev. Lett. 83,
1219 (1999).

${ }^{9}$ T. Stanescu, I. Martin, and P. Phillips, cond-mat/9905116 (unpublished).

${ }^{10}$ See review by G. Grüner, Rev. Mod. Phys. 60, 1129 (1988).

${ }^{11}$ See review by G. Nachtwei, Physica E (Amsterdam) 4, 79 (1999).

${ }^{12}$ See article by M. Shayegan in Ref. 1.

${ }^{13}$ V.J. Goldman, M. Santos, M. Shayegan, and J.E. Cunningham, Phys. Rev. Lett. 65, 2189 (1990).

${ }^{14}$ F.I.B. Williams et al., Phys. Rev. Lett. 66, 3285 (1991).

${ }^{15}$ Y.P. Li et al., Phys. Rev. Lett. 67, 1630 (1991).

${ }^{16}$ H.W. Jiang et al., Phys. Rev. B 44, 8107 (1991).

${ }^{17}$ S. Komiyama, T. Takamasa, S. Hiyamizu, and S. Sasa, Solid State Commun. 54, 479 (1985). 